University of Nebraska - Lincoln

DigitalCommons@University of Nebraska - Lincoln

Uniformed Services University of the Health

Sciences

U.S. Department of Defense

2011

\title{
P11 expression and PET in bipolar disorders
}

\author{
Lei Zhang \\ Uniformed Services University of the Health Sciences, Lezhang@USUHS.mil \\ Cheng-Ta Li \\ National Yang-Ming University \\ Tung-Ping Su \\ National Yang-Ming University \\ Xian-Zhang $\mathrm{Hu}$ \\ Uniformed Services University of the Health Sciences \\ Ruth A. Lanius \\ University of Western Ontario \\ See next page for additional authors
}

Follow this and additional works at: https://digitalcommons.unl.edu/usuhs

Part of the Medicine and Health Sciences Commons

Zhang, Lei; Li, Cheng-Ta; Su, Tung-Ping; Hu, Xian-Zhang; Lanius, Ruth A.; Webster, Maree J.; Chung, MingYi; Chen, Ying-Sheue; Bai, Ya-Mei; Barker, Jeffery L.; Barrett, James E.; Li, Xiao-Xia; Li, He; Benedek, David M.; and Ursano, Robert, "P11 expression and PET in bipolar disorders" (2011). Uniformed Services University of the Health Sciences. 68.

https://digitalcommons.unl.edu/usuhs/68

This Article is brought to you for free and open access by the U.S. Department of Defense at DigitalCommons@University of Nebraska - Lincoln. It has been accepted for inclusion in Uniformed Services University of the Health Sciences by an authorized administrator of DigitalCommons@University of Nebraska Lincoln. 


\section{Authors}

Lei Zhang, Cheng-Ta Li, Tung-Ping Su, Xian-Zhang Hu, Ruth A. Lanius, Maree J. Webster, Ming-Yi Chung, Ying-Sheue Chen, Ya-Mei Bai, Jeffery L. Barker, James E. Barrett, Xiao-Xia Li, He Li, David M. Benedek, and Robert Ursano 


\title{
P11 expression and PET in bipolar disorders
}

\author{
Lei Zhang $^{\mathrm{a}, *, 1}$, Cheng-Ta $\mathrm{Li}^{\mathrm{b}, \mathrm{c}, 1}$, Tung-Ping Su ${ }^{\text {b,c,1 }}$, Xian-Zhang Hu ${ }^{\mathrm{a}}$, Ruth A. Lanius ${ }^{\mathrm{d}}$, Maree J. Webster ${ }^{\mathrm{e}}$, \\ Ming-Yi Chung ${ }^{\mathrm{f}}$, Ying-Sheue Chen ${ }^{\mathrm{b}}$, Ya-Mei Bai ${ }^{\mathrm{b}}$, Jeffery L. Barker ${ }^{\mathrm{g}}$, James E. Barrett ${ }^{\mathrm{h}}$, Xiao-Xia Li $^{\mathrm{a}}$, \\ He Li ${ }^{\text {a }}$, David M. Benedek ${ }^{\mathrm{a}}$, Robert Ursano ${ }^{\mathrm{a}}$ \\ ${ }^{a}$ Center for the Study of Traumatic Stress, Department of Psychiatry, Uniformed Services University of the Health Sciences, Bethesda, MD 20814, USA \\ ${ }^{\mathrm{b}}$ Division of Psychiatry, Faculty of Medicine and Institute of Brian Science, National Yang-Ming University, Taipei, Taiwan \\ ${ }^{\mathrm{c}}$ Department of Psychiatry, Taipei Veterans General Hospital, Taipei, Taiwan \\ ${ }^{\mathrm{d}}$ Department of Psychiatry, Traumatic Stress Service, London Health Sciences Centre, The University of Western Ontario, London, ON, Canada \\ e Stanley Medical Research Institute, USA \\ ${ }^{\mathrm{f}}$ Life Sciences \& Institute of Genome Sciences, National Yang-Ming University, Taipei, Taiwan \\ ${ }^{\mathrm{g}}$ Laboratory of Neurophysiology, National Institute of Neurological Disorders and Stroke, National Institutes of Health, Bethesda, MD 20892, USA \\ ${ }^{\mathrm{h}}$ Department of Pharmacology and Physiology, Drexel University, College of Medicine Philadelphia, PA 19102-1192, USA
}

\section{A R T I C L E I N F O}

\section{Article history:}

Received 11 April 2011

Received in revised form

31 May 2011

Accepted 8 June 2011

\section{Keywords:}

p11

PET

Bipolar disorder-I

Bipolar disorder-II

Biomarker

Amygdala

\begin{abstract}
A B S T R A C T
Background: Bipolar disorder (BD) is a common mental disorder, subdivided into BD-I and BD-II. Currently, few biomarkers differentiate BD-I from BD-II. However, it is suggested that peripheral blood mononuclear cell (PBMC) mRNA levels of p11 and positron emission tomography (PET) might be potential biomarkers for BD.

Methods: Healthy controls (HCs), BD-I, and BD-II patients in remission ( $n=20$ in each group) underwent a resting PET study with the radiotracer $\left[{ }^{18} \mathrm{~F}\right]-2$-deoxy-2-fluoro-D-glucose $\left({ }^{18} \mathrm{~F}-\mathrm{FDG}\right)$. PBMC p11 mRNA levels were determined by quantitative real-time PCR.

Results: Comparing BD patients to HCs, normalized glucose metabolism (NGM) was higher in the hippocampus, parahippocampus, and amygdala, but lower in the anterior cingulate cortex (aCC), medial prefrontal cortex (mPFC), dorsolateral prefrontal cortex (dIPFC), insula and thalamus. Compared to BD-II, BD-I had hypometabolism of glucose in the aCC, bilateral middle and inferior gyrus, insula and striatum, and hypermetabolism of glucose in the left parahippocampus. PBMC p11 mRNA was over-expressed in both BD-I and BD-II, although there was no significant difference in its expression levels between BD-I and B-II patients. Further, there were significant positive correlations between PBMC p11 mRNA and NGM in the mPFC, aCC, left insula, bilateral orbitofrontal cortex (OFC), and left middle, inferior and superior temporal gyri. Also, PBMC p11 mRNA was positively correlated to the number of depressive episodes in BD patients, especially in BD-I patients.

Discussion: This study demonstrates that PBMC p11 mRNA expression is associated with neural activation in the brain of BD patients and warrants a larger translational study to determine its clinical utility.
\end{abstract}

Published by Elsevier Ltd.

\section{Introduction}

BD is a complex, chronic psychiatric condition characterized by recurring episodes of depression and mania or hypomania (Michalak et al., 2008). It is subdivided into BD-I and BD-II, based on the nature and severity of the mood episodes experienced. For a diagnosis of BD-I, patients must have one or more manic episodes, while a depressive or hypomanic episode is not required. For BD-II

\footnotetext{
* Corresponding author. Tel.: +1 301295 0921; fax: +1 3012950923.

E-mail address: Lezhang@USUHS.mil (L. Zhang).

1 Equal contribution.
}

diagnosis, patients must have one or more major depressive episodes and no manic episodes, but may have one or more hypomanic episodes. BD-II is sometimes difficult to distinguish from BD-I clinically since the hypomanic episodes may simply appear as a period of high productivity, and 'social or occupational impairment' is hard to define under certain circumstances (Salvadore et al., 2008). Furthermore, lacking objective criteria, the diagnosis of BD is based on a subjective description by the patient and a subjective observation by the psychiatrist. Although there have been efforts to develop a clinical strategy to diagnose BD, there is still no objective biological assay for the diagnosis of BD or for the differentiation of BD-I from BD-II. The lack of quantitative objective measures of $\mathrm{BD}$ is one reason that the causative factors of 
BD remain obscure. As it is believed that genetic factors and variability in neuronal activity contribute substantially to the likelihood of developing BD (Craddock et al., 2005), we investigated the correlation between PBMC p11 mRNA levels using real-time PCR and neuronal activity using PET with ${ }^{18} \mathrm{~F}$-FDG, in a group of $\mathrm{BD}$ patients in remission. Previously, we found that p11 mRNA was over-expressed in the PBMCs of patients with BD compared to normal controls. P11, also known as S100A10, is a protein encoded by the S100A10 gene in humans. It is a member of the S100 family of proteins containing two EF-hand calcium-binding motifs, and is localized in the cytoplasm and/or nucleus in a wide range of cells (Manev and Manev, 2006). P11 regulates a number of cellular processes such as cell cycle progression and differentiation. It is implicated in exocytosis and endocytosis by reorganization of F-actin (Gerke and Weber, 1985), as well as in the transport of the 5-HT1B receptor from the cytoplasm to the membrane (Svenningsson et al., 2006). An association between p11 and depression was found in a p11 knock-out study in mice and in a post-mortem study in depressed patients (Anisman et al., 2008; Svenningsson et al., 2006). Stress-induced changes in p11 expression most likely result from the activation of the hypothalamuspituitary-adrenal (HPA) axis (Anisman et al., 2008; Zhang et al., 2008), indicating that there may be relationships between peripheral p11 mRNA levels and CNS activity.

Using PET, recent studies found that BD patients have abnormal neural activity in the limbic-thalamic-cortical circuit (Salvadore et al., 2008). For example, medication-free depressed outpatients with bipolar disorder had decreased metabolism in prefrontal, anterior cingulate, insula, and ventral striatal areas (Brooks et al., 2009). BD patients in remission have increased neuronal activity in several limbic areas, such as hippocampus, parahippocampus and amygdala (Drevets et al., 2008). Visualization of the resting glucose metabolism in part of the prefrontal cortex (PFC), aCC, insula, and striatum can be used to distinguish subtypes of mood disorders (Brooks et al., 2009; Hosokawa et al., 2009).

Previous studies have reported that p11, a calcium binding protein, is associated with both depression (Svenningsson et al., 2006) and PTSD (Zhang et al., 2008). In the brain of patients, p11 mRNA was down-regulated in depression but up-regulated in PTSD. Although there is a large number of calcium binding proteins, p11 is the only one associated with depression and PTSD. These results led us to hypothesize that p11 might be a potential biomarker for BD. P11 regulates a number of cellular processes such as cell cycle progression and differentiation, is implicated in exocytosis and endocytosis by reorganization of F-actin, and interacted with and transported the 5-HT1B receptor from the cytoplasm to the membrane (Svenningsson et al., 2006). This suggests that p11's regulation of the 5-HT1B receptor is critical to mental status. P11 knock-out mice showed behavioral changes, which were similar to those of depression, and responded more poorly to antidepressant medication than wild type mice because of dysregulation of 5-HT1B receptor trafficking (Svenningsson et al., 2006). Antidepressant medication increased expression levels of p11 and reversed the depressive behavior (Svenningsson et al., 2006). Although PET provides direct evidence of changes in glucose metabolism, which may be used as a biomarker for BD diagnosis, the expense and radiation exposure limit its utility. Therefore, a blood biomarker for BD-I and BD-II, such as PBMC p11, may complement the PET strategy in routine clinical practice since analysis for mRNA markers in PBMCs is much less expensive than PET, and carries no risk of radiation exposure. The goal of the present study was to examine the hypothesis that PBMC p11 mRNA levels can serve as an adjunctive biomarker for $\mathrm{BD}$ and are correlated with neuronal activity, possibly indicating a role for $\mathrm{p} 11$ in the pathophysiology of BD.

\section{Methods and material}

\subsection{Ethics statement}

The study was performed in accordance with the Declaration of Helsinki and was approved by the Ethics Review Committee of the Taipei Veterans General Hospital. The study was described comprehensively to all subjects prior to their enrollment and all participants signed a written informed consent (registration number: R000003878; trial ID: UMIN000003199).

\subsubsection{Subjects}

The study subjects were outpatients of the Taipei Veterans General Hospital from 2007 February to 2010 May. The diagnoses were established by clinical history and by administration of the Mini International Neuropsychiatric Interview (MINI) as found in the Diagnostic and Statistical Manual of the American Psychiatric Association (4th Edition, 1994). Patients were recruited for the study only if they were 21-65 years of age, had no alcohol or substance abuse history, no major physical or neurological illness, and no comorbidity with schizophrenia, obsessive-compulsive spectrum disorders, or post-traumatic stress disorder. They received a range of medication based on their clinical presentation and clinician's judgment. To minimize heterogeneity, all participants were in the remission stage of BD (Hosokawa et al., 2009). A patient was considered to be in remission when, for at least 2 weeks, both the 17-item Hamilton Depression Rating Scales (HDRS17) score was 9 or less and the Young Mania Rating Scale (YMRS) score was 7 or less (Hirschfeld et al., 2007; Zimmerman et al., 2004). After MINI screening, a group of healthy controls $(n=20)$ also participated in the study for the ${ }^{18} \mathrm{~F}$-FDG PET comparisons. Those with a family history of an axis I disorder including, schizophrenia or major depression, in first degree relatives were excluded.

\subsection{PET study: imaging acquisition and analysis}

All 40 patients and 20 healthy subjects received PET scans. Patients fasted for at least $4 \mathrm{~h}$ before the PET examination. PET scans of glucose utilization were acquired on a PET/CT scanner (Discovery VCT; GE Healthcare, USA) with a 3D brain mode. PET images were acquired in 45 min after an intravenous injection of 370 MBq FDG. In the combined PET/CT imaging, the standard PET transmission scan is replaced by a low-dose CT scan. Then a CT-based attenuation correction is given. PET images were scanned in the resting state with eyes covered with a mask. The brain acquisition time was $15 \mathrm{~min}$. The system produces 47 consecutive slices over an axial length of $15.7 \mathrm{~cm}$ with a slice thickness of $3.75 \mathrm{~mm}$ and a transaxial FOV of $70 \mathrm{~cm}$. PET images were reconstructed in a $128 \times 128$ matrix using CT for attenuation correction with an ordered-subset expectation maximization iterative reconstruction algorithm (6 iterations and 14 subsets). Then the axial images were realigned to yield sagittal and coronal images.

\subsection{PBMC p11 mRNA assay}

The PBMC study involved 52 subjects, including patients with $\mathrm{BD}-\mathrm{I}(n=18)$, BD-II $(n=20)$, and normal controls $(n=14)$ from the Department of Psychiatry, Taipei Veterans General Hospital, Taiwan. Heparinized and unheparinized blood samples $(10 \mathrm{~mL}$ each) were collected by venipuncture in vacutainer tubes. PBMCs were separated by centrifugation on a Ficoll-Hypaque (Invitrogen) density gradient. The blood samples were stored at $-80{ }^{\circ} \mathrm{C}$. RNA was extracted from blood lysates using a PAXgen blood RNA validation Kit (PreAnalytiX, Qiagen, Valencia, CA). cDNA was generated 
Table 1

Demographic data of BD-I and BD-II patients.

\begin{tabular}{|c|c|c|c|c|}
\hline & \multicolumn{2}{|l|}{$\begin{array}{l}\text { BD-I } \\
n=20\end{array}$} & \multicolumn{2}{|l|}{$\begin{array}{l}\text { BD-II } \\
n=20\end{array}$} \\
\hline & Mean $/ n$ & $\mathrm{SD} / \%$ & Mean $/ n$ & $\mathrm{SD} / \%$ \\
\hline Male, $n(\%)$ & 10 & 50 & 6 & 29 \\
\hline Female, $n(\%)$ & 10 & 50 & 14 & 71 \\
\hline Age (yrs) & 43.9 & 2.6 & 42.9 & 2.8 \\
\hline Age at onset (yrs) & 31.5 & 2.4 & 31.3 & 2.4 \\
\hline Years of duration (yrs) & 12.7 & 2.7 & 11.5 & 1.5 \\
\hline $\begin{array}{l}\text { Number of depressive episodes } \\
\text { Medication }\end{array}$ & 3.9 & 3.7 & 6.4 & 5.1 \\
\hline Valproicacid (mg/day) & 798.1 & 238.1 & 768.1 & 248.6 \\
\hline Quetiapine (mg/day) & 245.6 & 161.6 & 213.3 & 83.3 \\
\hline Lamotrigene (mg/day) & 115.0 & 65.8 & 137.4 & 54.0 \\
\hline
\end{tabular}

from $3 \mu \mathrm{g}$ of total RNA using Superscript III RT (reverse transcriptase) and oligo (dT) primers (Invitrogen). Real-time PCR was performed on the generated cDNA product in the IQ5 sequence detection system using SYBR Green (Bio-Rad). The following sequences were used for human p11 mRNA analyses: forward 5'AAATTCGCTGGGGATAAAGG-3' and reverse 5'AGCCCACTTTG CCATCTCTA-3'primers. $\beta$-actin mRNA levels were used as an internal control for normalizing p11 mRNA levels in control and experimental samples. The sequences for $\beta$-actin primers were $5^{\prime}$ ACCTGTACGCCAACACAGTG-3' and 5'-ACACGGAGTACTTGCGCTCA$3^{\prime}$ (Applied Biosystems). Dilution curves confirmed the linear dependence of the threshold cycle number on the concentration of template RNAs. Relative quantitation of p11 mRNA in control and experimental samples was obtained using the standard curve method.

\subsection{Statistical analysis}

PET data were analyzed using the Statistical Parametric Mapping, version 2 (SPM2; Wellcome Department of Cognitive Neurology, Institute of Neurology, University College London, London, England) implemented in Matlab 7.0 (The Mathworks Inc., Sherborn, MA, USA). First, an ${ }^{18}$ F-FDG template was created for all subjects including all BD patients and HCs (Gispert et al., 2003). Then, each subject's images were normalized to the ${ }^{18} \mathrm{~F}$ FDG template and smoothed with a 3D Gaussian kernel $($ FWHM $=12 \mathrm{~mm})$. The overall grand mean from PET scans was centered and normalized to 100 , and global variance across scans was removed by analysis of covariance (ANCOVA) (Friston et al., 1990). To assess potential group differences of normalized brain glucose uptake, an ANCOVA with age, gender (Willis et al., 2002) and global gray matter values as covariates of no interest was used for between-group comparisons (BD-I vs. BD-II). The significance thresholds were set at $p<.05$ uncorrected at the voxel level, followed by a cluster-level analysis with a corrected $p<.05$. In addition, voxel-based partial correlations were performed for investigating the association between ${ }^{18} \mathrm{~F}$-FDG uptake and PBMC p11 mRNA levels after accounting for age, gender and total gray matter counts. Since the brain regions selected for study were those known to be involved in the neural system underlying BD - PFC, thalamus, aCC, insula, and striatum - the identified brain regions within a priori areas were further examined by Small Volume Correction (SVC) with an anatomically defined regional mask in the relevant gray matter area (Friston, 1997). Those brain regions passing through an SVCcorrected $p$-value $<.05$ were considered significant.
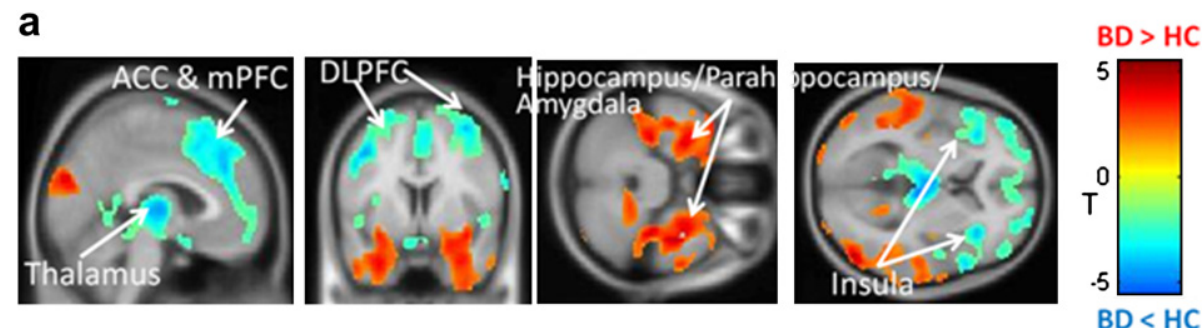

b
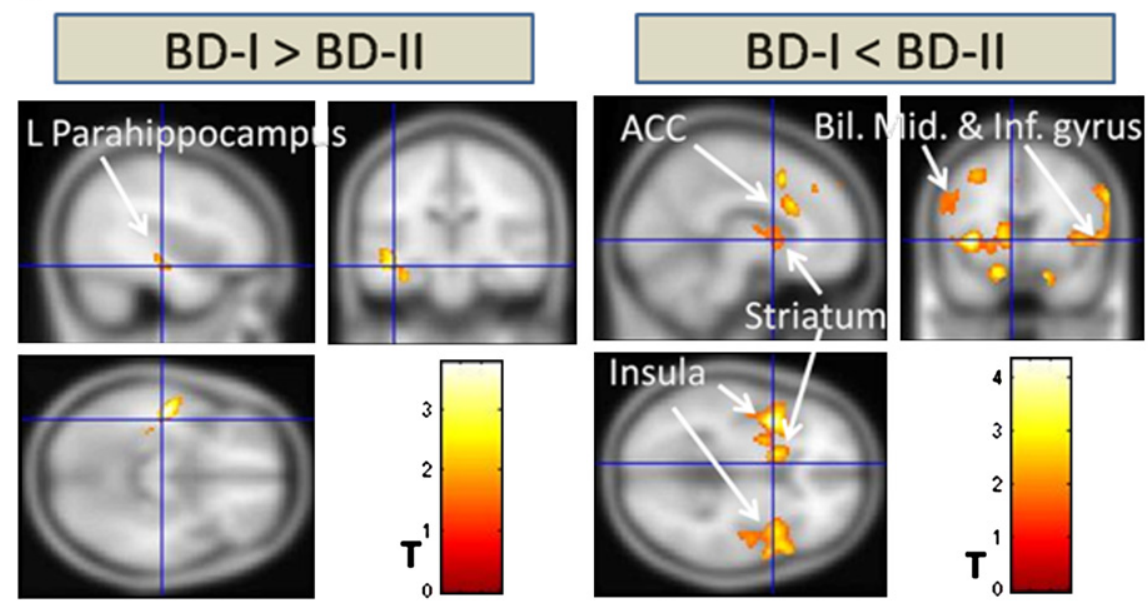

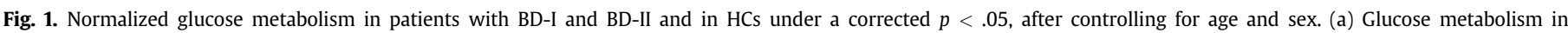

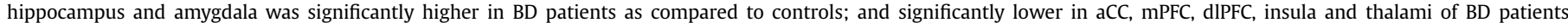

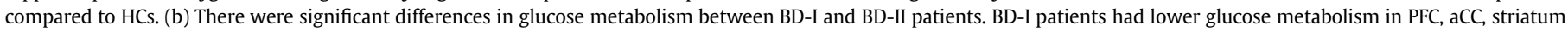
and bilateral insula (left panel), while BD-II patients demonstrated hypermetabolism of glucose in left parahippocampus/fusiform gyrus (right panel). 


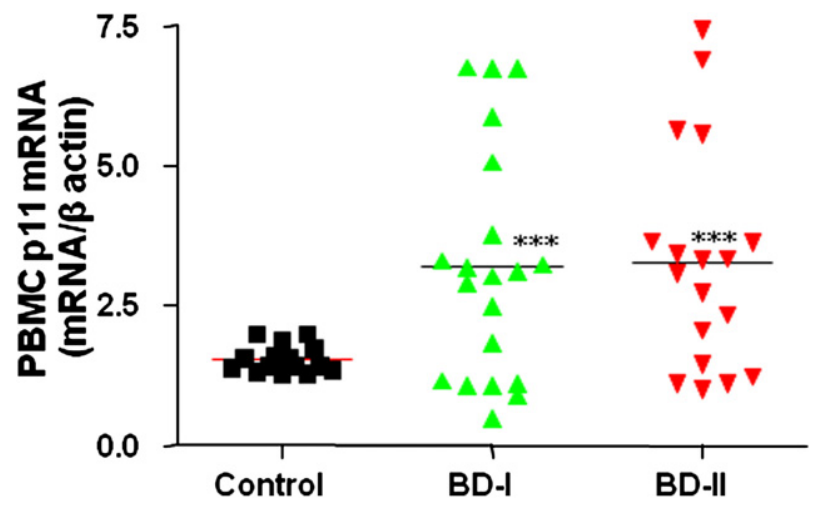

Fig. 2. PBMC p11 mRNA levels in BD-I and BD-II patients compared to HCs. Quantitative real-time PCR analysis revealed that p11 mRNA levels are significantly elevated in PBMCs of both BD-I and BD-II patients compared to HCs. There is no significant difference in PBMC p11 mRNA levels between BD-I and BD-II patients. ${ }^{* * *} p<.001$.

All PBMC p11 mRNA levels are presented as mean \pm S.D. Oneway ANOVAs were used to evaluate differences in PBMC p11 mRNA levels among groups of BD-I, BD-II and control subjects. The correlation coefficients and $p$-values were calculated using the GraphPad Prism (GraphPad Software, Inc. San Diego, CA).

SPSS 18.0 was used to perform linear regressive analysis to measure the correlation between the number of depressive episodes and PBMC p11 mRNA levels.

\section{Results}

In total, 40 right-handed patients (BD-I $=20, \mathrm{BD}-\mathrm{II}=20$ ) were in remission and did not differ with respect to current age, age at onset of $\mathrm{BD}$, gender, duration of illness, or number of previous depressive and manic episodes, and the dosage of medication the patients took (Table 1). NGM was significantly higher in BD patients as compared to $\mathrm{HCs}$ in the hippocampus, parahippocampus, and amygdala, and significantly lower in aCC, MPFC, dlPFC, insula and thalamic regions ( $p<.05$; Fig. 1a). As compared to BD-II patients, BD-I patients had hypometabolism of glucose in the aCC, bilateral middle and inferior gyrus, insula, and striatum and hypermetabolism of glucose in the left parahippocampus ( $p<.05$; Fig. $1 \mathrm{~b}$ ).
PBMC p11 mRNA levels were measured by quantitative realtime PCR (Fig. 2). P11 mRNA levels were significantly higher in the PBMCs of both BD-I and BD-II patients compared to HCs $\left({ }^{* * *} p<.001\right)$. However, PBMC p11 mRNA levels did not differ between BD-I and BD-II patients $(p>.05)$. P11 and normalized glucose metabolism were significantly and positively correlated in the PFC, aCC, left insula and left superior temporal gyrus (Fig. 3).

Linear regressive analysis showed that, in subjects with $B D$, PBMC p11 mRNA level was positively correlated with the number of depressive episodes $(r=0.38, p<.05)$ (Table 2). After further analysis, BD-I but not BD-II was found to be significantly correlated to p11 expression in PBMC (Table 2).

\section{Discussion}

There are five major findings in this study. First, we found that glucose metabolism levels in specific brain regions significantly differed between BD patients (both BD-I and BD-II) and HC's. Second, we examined the correlation between $\mathrm{p} 11$ expression and number of depressive episodes in BD, for both BD-I and BD-II. We found that the PBMC p11 mRNA levels were positively correlated with the number of depressive episodes in BD (Table 2). Further, this positive correlation was obtained in BD-I, but not in BD-II (Table 2). Third, we demonstrated that BD-I patients had significantly different glucose metabolism levels in a number of brain regions, as compared to BD-II patients. Fourth, we have shown that p11 mRNA was over-expressed in the PBMCs of both BD-I and BD-II patients, as compared to controls, although there was no significant difference in such expression levels between BD-I and BD-II. Finally, we found a positive correlation between p11 mRNA in PBMCs and glucose metabolism in the MPFC, aCC, left middle, left inferior, and left superior temporal gyri, left insula, and bilateral OFC in BD patients in remission.

The PBMC p11 mRNA over-expression might serve as a compensatory mechanism for the decreased neuronal activity in the brain of BD patients. Since the p11 protein is linked with the transport of 5-HT1B receptors from the cytosol to the membrane (Svenningsson et al., 2006) and has been implicated in the regulation of mood and stress through the HPA axis, these results are consistent with the hypothesis that the serotonergic system is involved in the pathophysiology of BD.

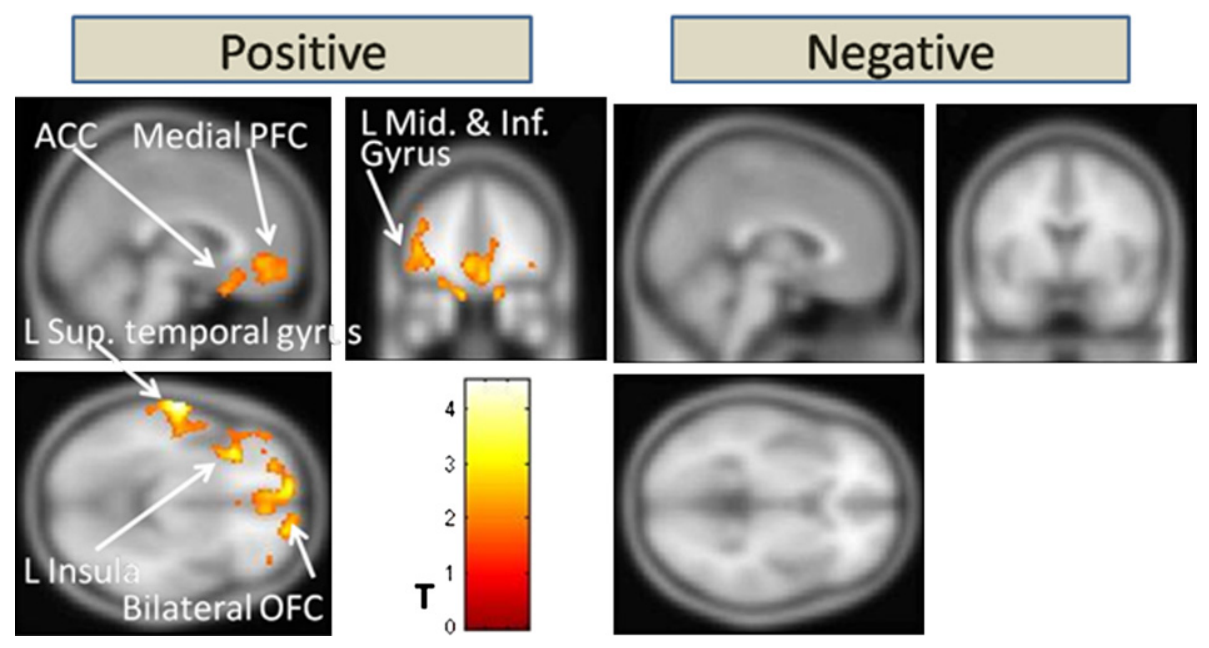

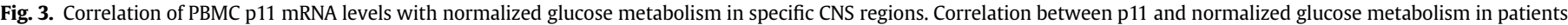

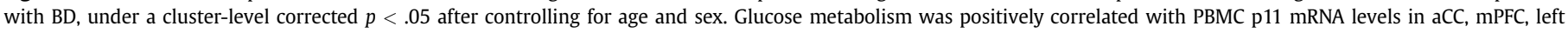
superior, middle and inferior temporal gyrii, left insula, and bilateral orbitofrontal cortex. 
Table 2

Linear regressive analysis of number of depressive episodes and p11 expression level.

\begin{tabular}{lllll}
\hline & B & r & t & $p$ value \\
\hline All $(N=40)$ & 2.58 & 0.38 & 2.45 & 0.02 \\
BD-I $(N=20)$ & 1.22 & 0.51 & 2.34 & 0.03 \\
BD-II $(N=20)$ & 2.81 & 0.24 & 1.03 & 0.31 \\
\hline
\end{tabular}

The over-expression of PBMC p11 mRNA in remission patients with BD may be caused by the dysregulation of certain brain regions, particularly the hippocampus and amygdala, in response to stress. A previous study on the stress-related regulation of p11 identified two specific glucocorticoid receptor response elements (GRE) in the p11 promoter region (Zhang et al., 2008). Stressinduced glucocorticoids activate glucocorticoid receptors (GR), which translocate from the cytosol to the nuclear membrane. In the nuclei, GR binds to the GRE in the p11 promoter and regulates p11 expression. This hypothesis was supported by our previous findings, which showed that either treatment with a GR antagonist or mutation of the GRE in the p11 promoter abolished glucocorticoidinduced p11 over-expression (Zhang et al., 2008). Thus, stressinduced p11 expression is mediated by GR and GRE in the p11 promoter, and this circuit may be involved in stress-related diseases such as PTSD and affective disorders. Several brain regions in which p11 is expressed (Sapolsky, 2000) are involved in the stress response. For example, evidence from functional imaging studies reveal that in anxiety disorders, brain activity in the amygdala is enhanced abnormally when patients are exposed to stressful situations (Drevets et al., 2002). Our findings of elevated neural activity in the hippocampus and other closely related brain regions, including the amygdala, could be a reflection of increased activity along the HPA axis, which has been implicated in the etiology of BD (Sassi et al., 2001). Even BD patients in remission present abnormal activity in these stress-related brain regions.

In this study, we demonstrated that the PBMC p11 mRNA levels were positively correlated with the number of depressive episodes of BD-I but not BD-II. Thus, PBMC p11 mRNA level may serve as a biomarker of BD-I. The effects of antidepressants on p11 expression levels in mice (Egeland et al., 2010) and glucose levels in human have been reported (Videbech, 2000). In the present study, we found there are no significant differences of medications between BD-I and BD-II groups. That observation may rule out the possibility that the differences of PBMC p11 mRNA levels between BD-I and BD-II were resulted from the differences of the administration of antidepressants. Although this needs to be validated using a larger sample size, such a biomarker would facilitate decision making by clinicians diagnosing and establishing a therapeutic strategy for BD.

To determine whether neural function can be a biomarker for differentiation of BD-I and BD-II, we compared PET results of the two groups. We found that, compared to BD-II patients, BD-I patients in remission exhibited hypometabolism in the aCC, bilateral middle and inferior gyrus, striatum and insula and hypermetabolism in the hippocampus. This result is consistent with previous studies investigating glucose metabolism in BD patients in remission (Brooks et al., 2009) and with our own unpublished data. In addition, PBMC p11 mRNA over expression was seen in both BD-I and BD-II, indicating that it might serve as a compensatory mechanism for the alteration of neuronal activity in the brain of BD patients.

The limitations of this study include limited sample size, lack of quantitative (absolute cerebral metabolism) data, and heterogeneous medication regimens in patients. To the best of our knowledge, however, this study is the first one to report a correlation between PBMC p11 mRNA expression and neural activation in the brain of BD patients. Our data warrant a comprehensive study to determine whether PBMC p11 expression can serve as a biomarker for BD diagnosis and whether PET can be a tool for differentiation of BD-I from BD-II.

\section{Contributors}

Lei Zhang made substantial contributions to the conception of the study, had the lead in the analysis and interpretation of the data, and wrote the manuscript.

Cheng-Ta Li, Ming-Yi Chung, Ying-Sheue Chen, Ya-Mei Bai, and Tung-Ping Su made substantial contributions to the sample collection, clinical observation, and data interpretation, and read critically for important intellectual content.

Xian-Zhang Hu and Xiao-Xia Li made substantial contributions to real-time PCR experiment and data collection. Jeffery L. Barker, James E. Barrett, He Li, Ruth A. Lanius, David M. Benedek and Robert Ursano made substantial contributions to interpretation of the data, and revised the manuscript critically for important intellectual content.

\section{Role of funding sources}

This research was supported in part by the Center for the Study of Traumatic Stress, Department of Psychiatry, Uniformed Services University of the Health Sciences and National Science Council, Taiwan (NSC 96-2752-B-010-006-PAE \& 97-2752-B-010-002-PAE).

\section{Conflict of interest}

The authors declare no conflict of interest.

\section{Acknowledgments}

We thank Francie Gabbay, Vivek Jayadeva and Stanley Smerin of USUHS for their assistance with editing the manuscript.

\section{References}

Anisman H, Du L, Palkovits M, Faludi G, Kovacs GG, Szontagh-Kishazi P, et al Serotonin receptor subtype and p11 mRNA expression in stress-relevant brain regions of suicide and control subjects. Journal Psychiatry Neuroscience 2008; 33:131-41.

Brooks 3rd JO, Wang PW, Bonner JC, Rosen AC, Hoblyn JC, Hill SJ, Ketter TA. Decreased prefrontal, anterior cingulate, insula, and ventral striatal metabolism in medication-free depressed outpatients with bipolar disorder. Journal of Psychiatric Research 2009;43:181-8.

Craddock N, O'Donovan MC, Owen MJ. The genetics of schizophrenia and bipolar disorder: dissecting psychosis. Journal of Medical Genetics 2005;42:193-204.

Drevets WC, Price JL, Bardgett ME, Reich T, Todd RD, Raichle ME. Glucose metabolism in the amygdala in depression: relationship to diagnostic subtype and plasma cortisol levels. Pharmacology Biochemistry and Behaviour 2002;71: 431-47.

Drevets WC, Savitz J, Trimble M. The subgenual anterior cingulate cortex in mood disorders. CNS Spectrums 2008;13:663-81.

Egeland M, Warner-Schmidt J, Greengard P, Svenningsson P. Neurogenic effects of fluoxetine are attenuated in p11 (S100A10) knockout mice. Biological Psychiatry 2010;67:1048-56.

Friston KJ. Testing for anatomically specified regional effects. Human Brain Mapping 1997;5:133-6.

Friston KJ, Frith CD, Liddle PF, Dolan RJ, Lammertsma AA, Frackowiak RS. The relationship between global and local changes in PET scans. Journal of Cerebral Blood Flow \& Metabolism 1990;10:458-66.

Gerke V, Weber K. The regulatory chain in the p36-kd substrate complex of viral tyrosine-specific protein kinases is related in sequence to the S-100 protein of glial cells. EMBO Journal 1985;4:2917-20.

Gispert JD, Pascau J, Reig S, Martinez-Lazaro R, Molina V, Garcia-Barreno P, Desco M. Influence of the normalization template on the outcome of statistical parametric mapping of PET scans. Neuroimage 2003;19:601-12.

Hirschfeld RM, Calabrese JR, Frye MA, Lavori PW, Sachs G, Thase ME, Wagner KD. Defining the clinical course of bipolar disorder: response, remission, relapse, recurrence, and roughening. Psychopharmacology Bulletin 2007;40:7-14. 
Hosokawa T, Momose T, Kasai K. Brain glucose metabolism difference between bipolar and unipolar mood disorders in depressed and euthymic states. Progress in Neuropsychopharmacology and Biological Psychiatry 2009;33:243-50.

Manev H, Manev R. Nomen est Omen: do antidepressants increase p11 or S100A10? Journal of Biomedical Discovery and Collaboration 2006;1:5.

Michalak EE, Murray G, Young AH, Lam RW. Burden of bipolar depression: impact of disorder and medications on quality of life. CNS Drugs 2008;22: 389-406.

Salvadore G, Drevets WC, Henter ID, Zarate CA, Manji HK. Early intervention in bipolar disorder, part I: clinical and imaging findings. Early Intervention in Psychiatry 2008;2:122-35.

Sapolsky RM. Glucocorticoids and hippocampal atrophy in neuropsychiatric disorders. Archives of General Psychiatry 2000;57:925-35.

Sassi RB, Nicoletti M, Brambilla P, Harenski K, Mallinger AG, Frank E, et al. Decreased pituitary volume in patients with bipolar disorder. Biological Psychiatry 2001 50:271-80.
Svenningsson P, Chergui K, Rachleff I, Flajolet M, Zhang X, El Yacoubi M, et al. Alterations in 5-HT1B receptor function by p11 in depression-like states. Science 2006;311:77-80.

Videbech P. PET measurements of brain glucose metabolism and blood flow in major depressive disorder: a critical review. Acta Psychiatrica Scandinavica 2000;101:11-20.

Willis MW, Ketter TA, Kimbrell TA, George MS, Herscovitch P, Danielson AL, et al. Age, sex and laterality effects on cerebral glucose metabolism in healthy adults. Psychiatry Research 2002;114:23-37.

Zhang L, Li H, Su TP, Barker JL, Maric D, Fullerton CS, et al. p11 is up-regulated in the forebrain of stressed rats by glucocorticoid acting via two specific glucocorticoid response elements in the p11 promoter. Neuroscience 2008;153:1126-34.

Zimmerman M, Posternak MA, Chelminski I. Derivation of a definition of remission on the Montgomery-Asberg depression rating scale corresponding to the definition of remission on the Hamilton rating scale for depression. Journal of Psychiatric Research 2004;38:577-82. 